\title{
MEDICO-GEOINFORMATON SYSTEM FOR SUICIDE PREVENTION AMONG ADOLESCENTS
}

\begin{abstract}
The development of adequate measures to combat the spread of socially significant diseases requires the use of modern tools available to demographers and epidemiologists for entering, analyzing and visualizing data on the spread of population movement processes in space. A medical and demographic geoinformation system has been created to study the dynamics of suicidal behavior in the Amur region of the Russian Far East. The main factors involved in the psychopathogenesis of suicidal behavior are considered. The research was conducted using various ecological and mathematical methods to assess the quality of life of the human population in extreme the conditions of the Far Amur region of the East of Russia, taking into account various factors of interaction between the individual, society and the environment. The features of the spread of suicidal attempts in the Amur region are revealed. The set of the main causes and factors of low intensity characterizing the current suicidal situation in the Amur region of the Russian Far East is studied on the example of the Khabarovsk city. The medicaldemographic and socio-psychological problems of suicidal behavior caused by depressive disorders are analyzed from the standpoint of the geoinformation modeling method. Original methodological approaches to the use of information models for medical-demographic and socio-economic studies of depressive spectrum disorders and the prevention of adolescents suicidal behavior are proposed.
\end{abstract}

KEYWORDS: medico-ecological geoinformaton system (MEGIS), cartography, suicidal behavior, depressive disorders (DD), adolescents, the Far East, Russia.

\section{INTRODUCTION}

The growing social significance of the damage caused by depressive disorders in recent decades, as well as real hopes for success in the fight against suicidal behavior, were the main reasons that retain scientific interest in DD [Bostic et al., 2005; Carrellas et al., 2017; Krasnov, 2011; Kripke, 1998; Malone et al., 2013]. Today, suicides occupy one of the leading places in the structure of premature mortality of the population, and their maximum frequency falls on the young and socially active age of the population ${ }^{3}$. The relevance of the problem of DD, which are increasingly manifested already in adolescence, is determined by their wide prevalence, the difficulty of recognizing this pathology in the early stages of the disease ${ }^{4}$, and the high risk of suicide [Clayton et al., 2013; Gladstone et al., 2009; Jinnin et al., 2016; Kretinin, 2015].

\footnotetext{
1 Pacific National University, Tihookeanskaya str., 136, 680035, Khabarovsk, Russia, e-mail: savin.sergei@mail.ru

2 Pacific National University, Tihookeanskaya str., 136, 680035, Khabarovsk, Russia, e-mail: kosyh.n@bk.ru

3 The Morbidity and Mortality Weekly Report (MMWR). 2020. № 69. P.1049-1057.

$4 \quad$ World Health Organization. Preventing suicide: A recourse for media professionals. Department of Mental Health Social Change and Mental Health. Geneva. 2014. 88 p.
} 
In most economically developed countries, depression ranks first or second among the causes of mental illness, and about a million people die from suicide every year in the world ${ }^{3}$. Suicide is a major public health concern. Over 48000 people died by suicide in the United States in 2018: it is the 10th leading cause of death overall. Suicide is complicated and tragic, but it is often preventable. Knowing the warning signs for suicide and how to get help can help save live [Carrellas et al., 2017; Gladstone et al., 2009; Krasnov, 2015; Stewart et al., 2010; Wasserman et al., 2017].

From 2015 geographic information system will be launched in Australia, which will collect data on male suicides for 6 years. GIS was developed by specialists from the Turning Point Drug and Alcohol Center at Monash University as part of a project launched by the Movember Foundation charity ${ }^{1}$. It will be the only geographic information system in the world that will try to collect statistics on the types of suicides and their geographical distribution. According to the authors of the project, a detailed analysis will help to identify problem regions and previously unknown trends. The GIS developed at the Turning Point center will focus on cases of male suicides, since men are less likely to seek psychological help than women, and at the same time they are 3 times more likely to die as a result of suicide. For example, in Victoria, the most populous state in Australia, out of 50 thousand calls to the ambulance, about $10 \%$ are incidents related to mental health. Of the victims, $40 \%$ are men, and two-thirds of the cases are suicides. GIS will collect accurate data on cases of male suicide, including geographical coordinates, type of incident, information about the victim (without specifying the name). According to experts of the Movember Foundation, the system will help to accurately identify the regions where the greatest number of suicides occurs, and the groups of people at greatest risk.

Russia continues to occupy one of the leading places in terms of the rate of teenage suicides [Kretinin et al., 2015; Oorzak, 2012], which in the future may lead to a significant increase in the number of mentally ill people in a number of territories. According to WHO, the Russian Federation ranks 1st in Europe in the number of suicides among adolescents ${ }^{2}$. The greatest frequency of suicides is observed in adolescents in the age range of 15-19 years and young people of 19-29 years [Churkin et al., 2009; Demcheva, 2021; Kretinin et al., 2015; Loginov et al., 2019]. In this category of suicides, there are up to 200 unrealized suicide attempts per 1 death [Shipitsyna et al., 2000]. Repeated suicidal acts are committed by about a third of suicides [Kretinin et al., 2015]. It was found that only $10 \%$ of adolescents in the case of suicide have a true desire to commit suicide, the remaining $90 \%$ of suicidal actions are demonstrative ${ }^{3}$.

The prevalence of depressive disorders that provoke suicide in prepubescent children is $1-2 \%$, and in children aged $8-13$ years, the risk of relapse is $40 \%$ within 2 years and $72 \%$ within 5 years [Krasnov, 2011; Kretinin et al., 2015; Loginov et al., 2019; Syrokvashina, 2017]. With age, the prevalence of suicidal depression in adolescents increases and is $3-8 \%$, and by the end of puberty reaches about 20\% [Oorzak, 2012; Syrokvashina, 2017]. Thus, the epidemiological situation with the spread of anxiety-phobic disorders and suicidal depression in recent decades reflects the general trends of tension in the world as a whole and in the Russian Far East, in particular, but significantly exceeds them in terms of the growth rate of mental illness and the number of registered patients, especially among the younger generation and students.

\footnotetext{
1 Addicted Australia, Turning Point. Available at URL: https://www.turningpoint.org.au/ (accessed 23.2.2021).

$2 \quad$ WHO Mortality Database. World Health Organisation Mortality Database. World Health Organization. Available at URL: https://www.who.int healthinfo_mortality (accessed 13.2.2021).

3 WHO. Depression: let's talk. 2017. Available at URL: https://www.who.int/news-room/detail/30-03-2017 (accessed 13.2.2021).
} 
The aim of the work is to identify the possibilities of geoinformation systems in the study of the causes and risk factors of suicidal behavior of adolescents of the Amur region suffering from disorders of the depressive spectrum.

\section{MATERIALS AND METHODS}

To solve these problems, the following research methods were used: medicalgeoinformation, medical-psychological, clinical-social, clinical-psychological, mathematicalstatistical. The program of interdisciplinary medical-psychological, clinical-social, clinicalpsychological research included the stages of collecting medical-psychological material (20132017); the stage of data processing (2017-2019); the stage of analyzing the results of the study (2018-2020). At the first stage of the study, primary scientific material was collected according to the inclusion criteria based on medical-psychological, socio-demographic (gender-age) and clinical features. At this stage, a complete clinical-psychopathological, clinical-sociopsychological examination of all patients hospitalized for non-psychotic DD in the hospital was conducted. After the initial examination, each patient underwent clinical follow-up until discharge or completion of therapy. At the second stage, statistical processing of the received materials was carried out. At the third stage, a clinical analysis, interpretation of the results obtained, and their comparison with the available literature data were carried out. The results were also summarized and the conclusions of the study were formulated, including specific recommendations for the differentiated prevention of pre-suicide DD and the prevention of repeated episodes of this psychopathology. The clinical-anamnestic method was used in the study of sociodemographic and socio-cultural characteristics of the subjects. These characteristics were determined based on a survey of patients and their immediate microsocial environment, as well as medical records. The use of the clinical and epidemiological method made it possible to identify and rank the frequency of occurrence of the identified risk factors. The epidemiological study was conducted by two methods: by clinical analysis of past life events and establishing their significance in the occurrence and development of pre-suicide DD (a retrospective method) and by direct clinical examination of patients aimed at identifying factors of etiological and pathogenetic significance. The results of the study were obtained during a clinical and psychopathological examination of 62 adolescents with non-psychotic DD living in Khabarovsk, who were admitted to the Khabarovsk Regional psychiatric Hospital after an incomplete suicide attempt. The final clinical and psychopathological examination was completed by 50 adolescents with non-psychotic depression who were undergoing inpatient treatment in psychiatric hospitals in Khabarovsk. The diagnosis of non-psychotic DD was based on criteria based on the ICD-10 classification [Loginov et al., 2019]. The Basic Map of Ethnocultural Studies developed at the "SSC of Social and Forensic Psychiatry", including sociodemographic, ethno-cultural and clinical topics, was used as a research tool for V.P. Serbsky [Churkin et al., 2009; Loginov et al., 2019]. The card was filled in for each subject during a personal clinical interview, supplemented with medical records (outpatient records, medical history) and information from the patients' next of kin. groups of patients meeting the following inclusion criteria: the presence of established clinical manifestations and maintenance of presuicide DD with clearly identifiable stress (traumatic) factor acute or prolonged nature; mental level DD; compliance with the clinical picture of the disorder one of the following diagnostic partitions (ICD-10): intentional self-harm (X60-X84); depressive episode of mild and moderate severity (F32.0, F32.1); dysthymia (F34.1); mixed anxiety and depressive disorder (F41.2); adjustment disorder (F43.20-22); the absence of signs of mental illnesses of other etiologies and exacerbations of chronic somatic diseases; the presence of informed consent of the patient or his legal representative to participate in the study. Additional sources of information were conversations with relatives and friends of patients, during which it was possible to obtain infor- 
mation about their behavior, statements, psychotraumatic situations, and circumstances. A study of available medical records (previous medical records and outpatient records of general medical institutions and specialized psychiatric institutions) was conducted, from which information on subclinical and clinical manifestations of pre-suicide DD could be extracted. The analysis and generalization of the data obtained from these sources, in most cases, allowed us to obtain fairly reliable information about the features of the clinical manifestations of each individual patient with suicidal tendencies. All patients underwent both a clinical and a special examination in accordance with the assigned tasks. The dynamics of depression was studied depending on the degree of decline in the corresponding subscales. These methods were chosen taking into account the availability of their implementation, as well as the possibility of a comprehensive assessment of verbal and non-verbal signals of personal access, which is especially important when working with depressed patients. It should be noted that the clinical diagnosis of presuicide DD had its own characteristics. So, in a conversation with many patients, it was important to take into account such features as a tendency to disguise, conceal their experiences, which made it difficult to make initial contact and required skills to quickly establish contact with the patient, the ability to inspire confidence in him. An important role was played by the observation of the patient during the interrogation: facial expressions, movements, speech, and the manner of self-control demonstrated his objective mental state. It is also important to talk to relatives, from whom you can get objective information about the patient's condition and the development of subclinical and clinical manifestations. To solve medical and geographical problems, various approaches were used to build information and computing systems of complex living objects based on multi-layer spatial models, primarily geoinformation systems, where the problems of combining and manipulating graphical and attribute data within a single integrated model were solved to a certain extent [Kosykh et al., 2008]. For the tasks of psychiatry and, in particular, suicidology, appropriate tools for visualization and descriptive analysis have been adapted and created. The Far East, with its unique population (aborigines and immigrants), a wide range of climatic and biogeochemical conditions, and a unique demographic situation, is a unique testing ground for conducting socio-psychological, epidemiological, and medicodemographic studies using modern geoinformation technologies. Developed and adapted MEGIS databases for monitoring the mental health of the population of the Amur region, computer atlases for different spatial layers that determine the suicidal situation in the region. Built-in geoinformation technologies and other mathematical tools for processing medical demography data were used: correlation and regression analysis, multivariate analysis of low-intensity factors (LIF) analysis, etc.

\section{RESULTS AND DISCUSSION}

As a result of using the main tool for a comprehensive study - a formalized "Statistical survey map", designed for the study of suicidal depressive disorders in adolescents, reliable data were obtained for the formation of a database of an information-recognition system on a cartographic basis - medical-ecological GIS (MEGIS). The map included 64 questions, arranged in the following sections: anamnestic information, sociodemographic, clinical, social, ethnocultural; forms and methods of interventional and post-interventional influences. Each point on the map reflected a specific the patient and contained several answer options that it was necessary to indicate when filling out. Additional sources of information were conversations with relatives of teenagers, during which it was possible to obtain objective data on behavioral disorders, statements, traumatic situations and circumstances. In addition, the available medical documentation was studied and entered, from which it was possible to obtain information about subclinical documents. and clinical manifestations of depressive disorder. Analysis and synthesis 
information obtained from the listed sources, in most cases provided objective information about the clinical manifestations of pre-suicide DD specifically for each teenager.

The dynamics of depression have been studied according to the degree of reduction of the indicators of the respective subscales. These methods were selected taking into account the availability of their conduct, as well as the possibility of a comprehensive assessment of verbal and nonverbal signals of personal access, which is especially important when working with depressed patients. It should be noted that the clinical diagnosis of pre-suicide DD had its own characteristics. So, in conversation with many patients, it was important to take into account such features as the tendency to dissimulate, hide their experiences, which made it difficult to primary contact and required skills in quickly establishing contact with the patient, the ability to inspire confidence in him. An important role was played by the observation of the patient during questioning: facial expressions, movement, speech, manner of self-control demonstrated his objective mental state. It is also important to talk with relatives, from whom it was possible to obtain objective information about the patient's condition and the development of subclinical and clinical manifestations. At the first stage of research, primary scientific material was collected based on inclusion criteria based on medico-psychological, socio-demographic (gender and age) and clinical features. At this stage, a complete clinical, psychopathological, clinical, social and psychological examination of all patients hospitalized for non-psychotic DD in hospital was carried out. After the initial examination, each patient was followed up with clinical follow-up until discharge or completion of therapy. At the second stage, statistical processing of the received materials was carried out. At the third stage, clinical analysis, interpretation of the results, their comparison with the existing literature data were carried out. Also, the results were summarized and the conclusions of the study were formulated, including specific recommendations for the differentiated prevention of pre-suicide DD and prevention of repeated episodes of this psychopathology. Clinical and anamnesis method was used in the study sociodemographic and socio-cultural characteristics of the subjects. These characteristics were determined on the basis of a survey of patients and their immediate microsocial environment, as well as medical records. The use of clinical and epidemiological method allowed to identify and rank the frequency of occurrence of the identified risk factors. The epidemiological study was carried out by two methods: by clinical analysis of past life events and establishing their significance in the emergence and development of pre-suicide DD (retrospective method) and by direct clinical examination of patients aimed at identifying factors of etiological and pathogenetic significance.

The study of the psychopathological structure of hereditary burden among the respondents ' relatives showed that most often the relatives of adolescents suffered from mental disorders associated with the use of psychoactive substances (alcoholism) - 32\%. DD with suicidal burden had $4 \%$, personality and behavior disorders $2 \%$. Almost every fourth teenager came from the so-called "alcoholic" family, which is consistent with the data [Loginov, 2019] that children from "alcoholic" families risk of depression is 7 times higher than children from "normal" families. Less often in close relatives there were other mental disorders: depressive disorders (suicidal burden) $-4 \%$, personal $-2 \%$. In most cases $(32 \%)$ the burden was associated with the use of psychoactive substances. The revealed distribution shows that not only mental disorders of the endogenous level (they were about 5\%), but also disorders of the exogenous level contribute to the genetic formation of pre-suicide DD, which once again emphasizes the importance of psychosocial factors in the Genesis of non-psychotic depressive disorders. From the standpoint of hereditary burden, the greatest risk of developing DD are persons whose relatives suffered from disorders associated with alcohol consumption, and the presence of psychopathological and suicidal burden of heredity in General can be considered as a significant factor in the development of depression. Continuous clinical-epidemiological and clinical- 
psychopathological study of persons suffering from pre-suicide DD and hospitalized for this reason in the Department of the Regional psychiatric hospital of Khabarovsk allowed to identify the most common clinical forms of non-psychotic depression among adolescents living in crossborder areas. In adolescents of the second age group (15-18 years) the clinical and psychopathological structure of DD was significantly more often directed towards the growth of short-term (42\%) and prolonged (24\%) depressive reactions. Among adolescents reliable data on the prevalence of a particular syndrome was not revealed. Such experiences provoked the appearance of a chronic experience of anxiety (if the situation was associated in the consciousness of the patient with a bad, in his opinion, the attitude of others) or anguish (if there were persistent ideas of their own uselessness, most often to their loved ones). For a better understanding of the influence of clinical factors, we have studied some features of the attitude to alcohol in adolescents suffering from pre-suicide DD. We took into account the subjective assessment of the patients themselves, so the results may have errors due to fear of disclosure of information to parents. The use of alcoholic beverages within the cultural norm was recorded in more than half of the adolescents, due to the ethnic characteristics of the country and the attitude to alcohol in the family.

The study of the features of the territorial distribution of various diseases, including presuicidal depression (DD), is difficult to carry out without the involvement of means of medical and geographical zoning. In the most general sense, medico-geographical zoning is an artificial division of the territory into a number of areas, areas (zones) that differ in certain parameters that can have a certain impact on the risk of the disease under study. At the same time, the options for dividing the same territory into zones may be different and depend on the form of the psychotic disease studied using the method of medico-geographical zoning [Kosykh, 2008]. The territorial position of the selected zone itself, in addition to the factors taken into account in this division of the territory, provides various additional information about the factors that are present in this zone due to its geographical location. Such additional information may include natural, demographic, social, and other factors. These factors must be taken into account when explaining the features of the spread of the studied disease in the selected areas. A similar method of zoning was used to study the spread of pre-suicide DD in children in a large-scale and climatically-geographically heterogeneous region of the Russian Far East. The assessment of the territorial distribution of pre-suicide DD is impossible without determining the minimum unit of observation-the taxon. Such taxons can be neither regions, nor territories, nor administrative districts for a number of reasons. The territories of the territories and regions of the Far East are extremely large, and many of them are located in several climatic zones. Some areas that are part of the territories and regions are populated unevenly and are characterized by varying degrees of economic development. Administrative districts, although more homogeneous in terms of economic development, are usually characterized by a low population density. This makes it somewhat difficult to determine a reliable picture of the spread of such a rare phenomenon, such as, for example, suicide after DD in children of the region. Thus, the optimal taxon is not a largescale region or a district with a small population, but a group of nearby administrative districts.

The question arises: the similarity of which parameters should be the basis for combining the districts? As it was shown in [Loginov et al., 2020], the risk of suicide after DD occurrence is influenced to some extent by some natural-climatic, socio-economic and environmental factors. In these conditions, the definition of the principle of zoning of the Far Eastern region is at the first stage the task of ranking the above factors according to the degree of their influence on each other. Discriminant analysis shows the correctness of this division when studying the features of the territorial distribution of a number of pre-suicide DD [Kosykh et al., 2008; Loginov et al., 2020]. Thus, the problems of medico-geographical zoning can be solved by methods of multidimensional statistics using cluster-discriminant analysis. When conducting zoning, it is 
necessary to adhere to the following principles: the minimum unit of observation should be a group of administrative districts that are similar in terms of parameters that presumably affect the characteristics of the spread of pre-suicide DD; the qualitative parameters should be translated into quantitative ones; the coordinates of administrative districts should be included in the general matrix as independent variables. The most promising method in mapping problems is the information game-theoretic modeling method [Polumienko et al., 2007], which, unlike the wellknown methods of zoning territories, uses a combination of the mathematical apparatus of game theory and the theory of pattern recognition. So MEGIS differs from the information retrieval system described above in the introduction ${ }^{5}$, which is an information recognition system based on the concept of information modeling, according to which all available information about the object of research is collected and accumulated, and then the recognition problem is solved. In turn, information-recognition systems solve the problem of pattern recognition as a problem of calculating properties [Polumienko et al., 2007].

To study the influence of socio-economic human ecology and mental factors and on the peculiarities of the territorial distribution of suicides, including advanced forms of DD, a population-epidemiological study was conducted in the Khabarovsk city. It was believed that the study of relationships between socio-economic and demographic and mental health characteristics of the individual administrative districts, on the one hand, and population frequency of a particular disease, on the other hand, can be an evidence of the social aspects of diseases. The methodological basis of the study was the analysis of multiple linear regression with an assessment of the contributions of the studied factors to the formation of the output parameter [Polumienko et al., 2007; Kosykh et al., 2008].

The problems of combining and manipulating graphical and attribute data within a single integrated model that arise in medical and environmental studies are solved by means of tool packages of geographical information systems (MEGIS). MEGIS is based on a spatial model consisting of several thematic layers. Each graphic object in the layer has its own attribute description, which is stored in an embedded or external database. In this case, the layers of the spatial model can be any subsystems of a complex ecological object (system of objects).

A methodology for creating geographic information systems in Khabarovsk has been developed, including data on the radon hazard of areas designated for residential development, the radon hazard of individual buildings, territorial characteristics of soil contamination with heavy metals, the location of power lines and highways with heavy traffic, and other harmful environmental factors. The geographic information system of the city includes a map of the territorial distribution of cases of suicides, the most important socio-economic and medical-geographical characteristics of administrative districts.

Methodological approaches to assessing the risk of suicides and DD, depending on the place of residence, have been developed. An appropriate software and hardware complex is being developed to solve the problems of medical ecology through GIS technologies and environmental monitoring. The possibility of applying a single methodological approach for geoinformation modeling of the spread of various forms of DD is justified.

According to its epidemiological characteristics, anthropogenic pollution of the environment, primarily the atmosphere, can be attributed to low-intensity factors (LIF). GIS is a way to study the environmental situation in the territories. Therefore, in the problems of psychoepidemiology, MEGIS can be a method of studying LIF, cluster and discriminant analyses can be used in complex mathematical support of geoinformation systems.

A feature of MEGIS is the principles of organizing an information system, allowing to take into account the presence of a person on a sufficiently large territory and analyze the likelihood of its presence in different parts of a given territory. The territory that is visited with the 
greatest frequency by a person during a certain the period of time is defined by us as the "vital activity zone" (VAZ). The principle of the formation of healthy life activity is shown in Fig. 1.

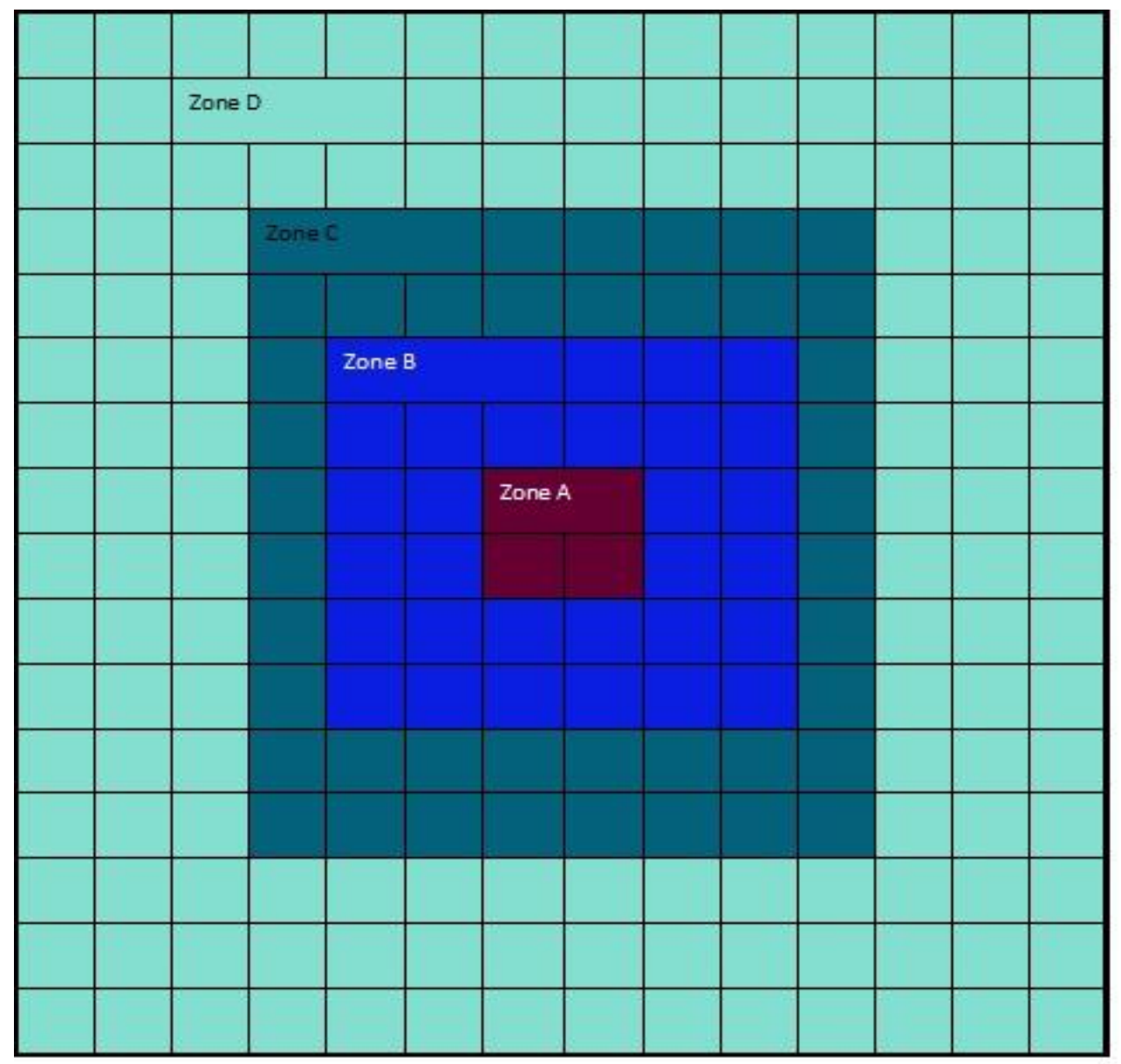

Fig. 1. Conditional scheme of human vital activity zones

In Fig. 1, the territory visited by a person during the conditional time is represented by a square consisting of zones $\mathrm{A}, \mathrm{B}, \mathrm{C}$ and $\mathrm{D}$. We consider that in zone $\mathrm{A}$ he spends up to $50 \%$ of the conventional time, in zone $\mathrm{B}-30 \%$, in zone $\mathrm{B}-15 \%$, and in zone $\mathrm{D}-5 \%$. The entire territory of the conditional square will be VAZ.

When constructing MEGIS, this territory can be taken into account by us in full, or only its individual parts are taken into account. So knowing that in zones A, B and C a person spends most of the conditional time (95\%), these territories will be considered by us as VAZ.

The possibility of applying the VAZ principle in MEGIS can be realized using the methods of enlarging squares or extrapolating information to the center of a square. The squares enlargement method is implemented by dividing the city territory into squares $1 \mathrm{~km} \mathrm{x} 1 \mathrm{~km}, 2 \mathrm{~km} \mathrm{x}$ $2 \mathrm{~km}$ and larger. In these large squares, not only mortality rates are determined, but also the average values of indicators of anthropo-technogenic pollution of the environment. And cartograms, divided into large squares, a spreadsheet is formed according to the principles that were considered earlier in the impact of certain environmental factors on a person is studied in a fairly limited area (usually within the city). 
So, a person is not a static unit of observation. During a certain period of time individual makes movements within a certain territory (zones), which has different social and ecological environmental characteristics in different parts of it. At the same time, the intensity of the impact of environmental factors on a person in different parts of the territory will be different (Fig. 2). This feature of the object of observation is implemented in MEGIS by methods of cell enlargement or extrapolation of information to the center of the square [Kosykh et al., 2008].

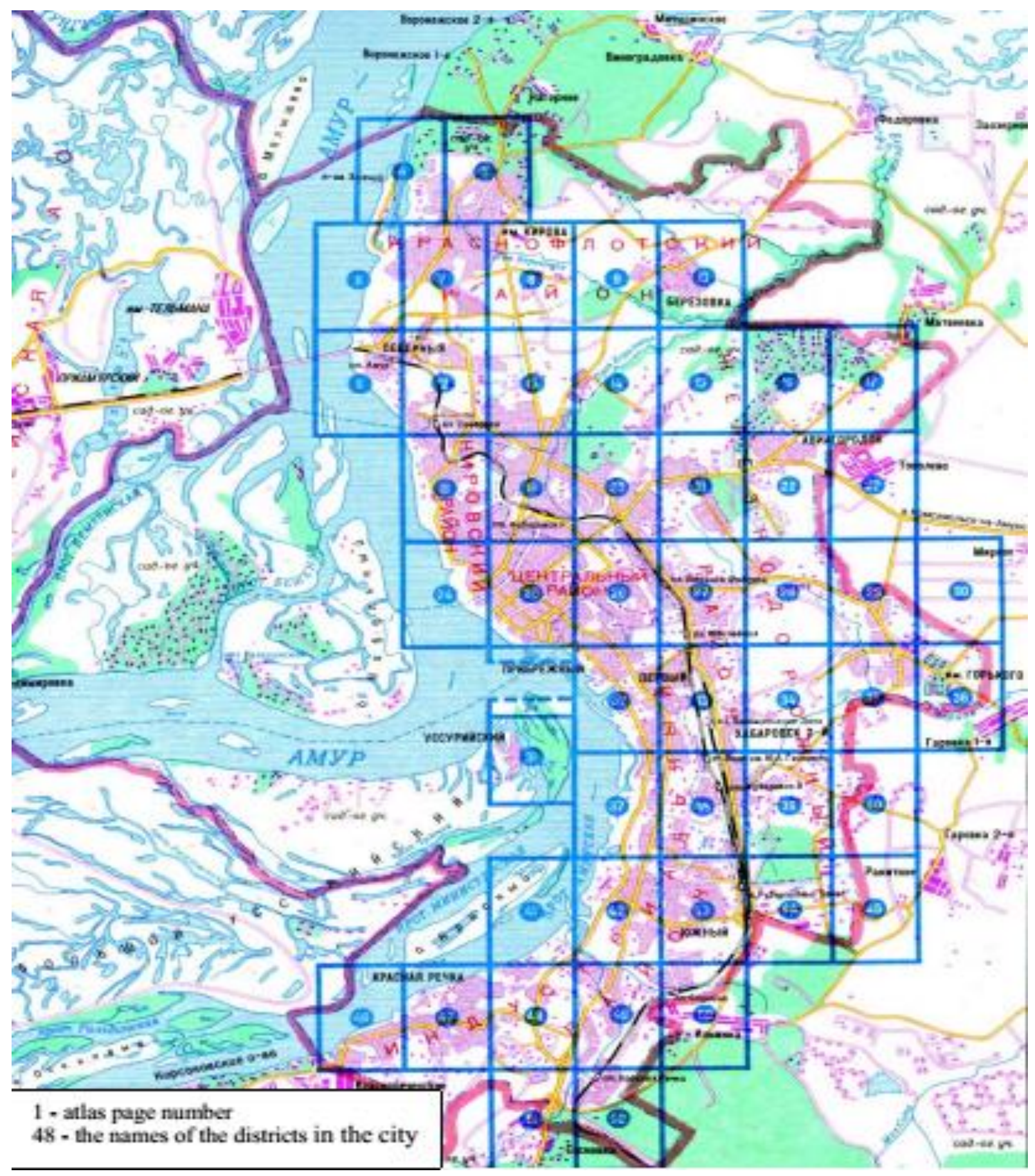

Fig. 2. An example of partitioning the territory of Khabarovsk using the Geoplex program 
On the basis of these principles, a special type of GIS - MEGIS was theoretically justified and created. This geographic information system was built on the basis of a series of cartograms of the city of Khabarovsk, reflecting the spread of deaths from suicides and some environmental parameters of the city's air basin (Fig. 3).

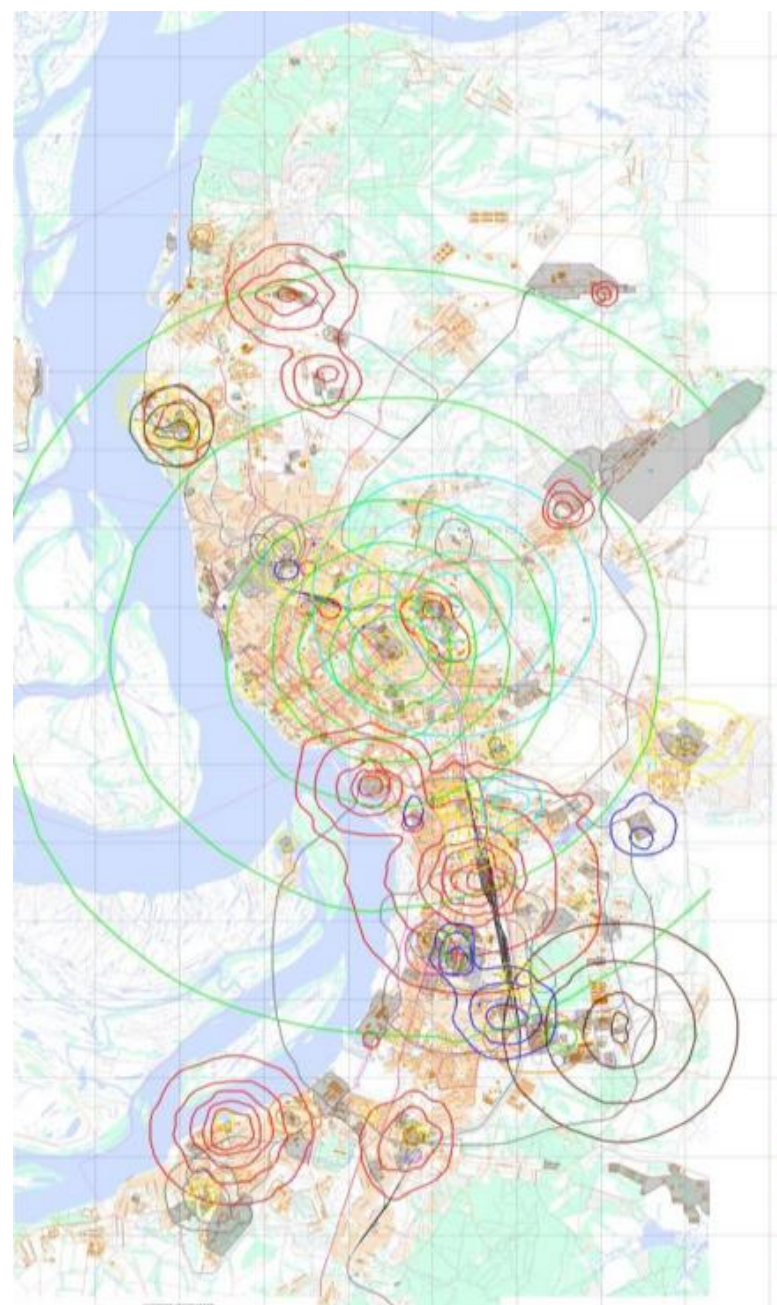

2003

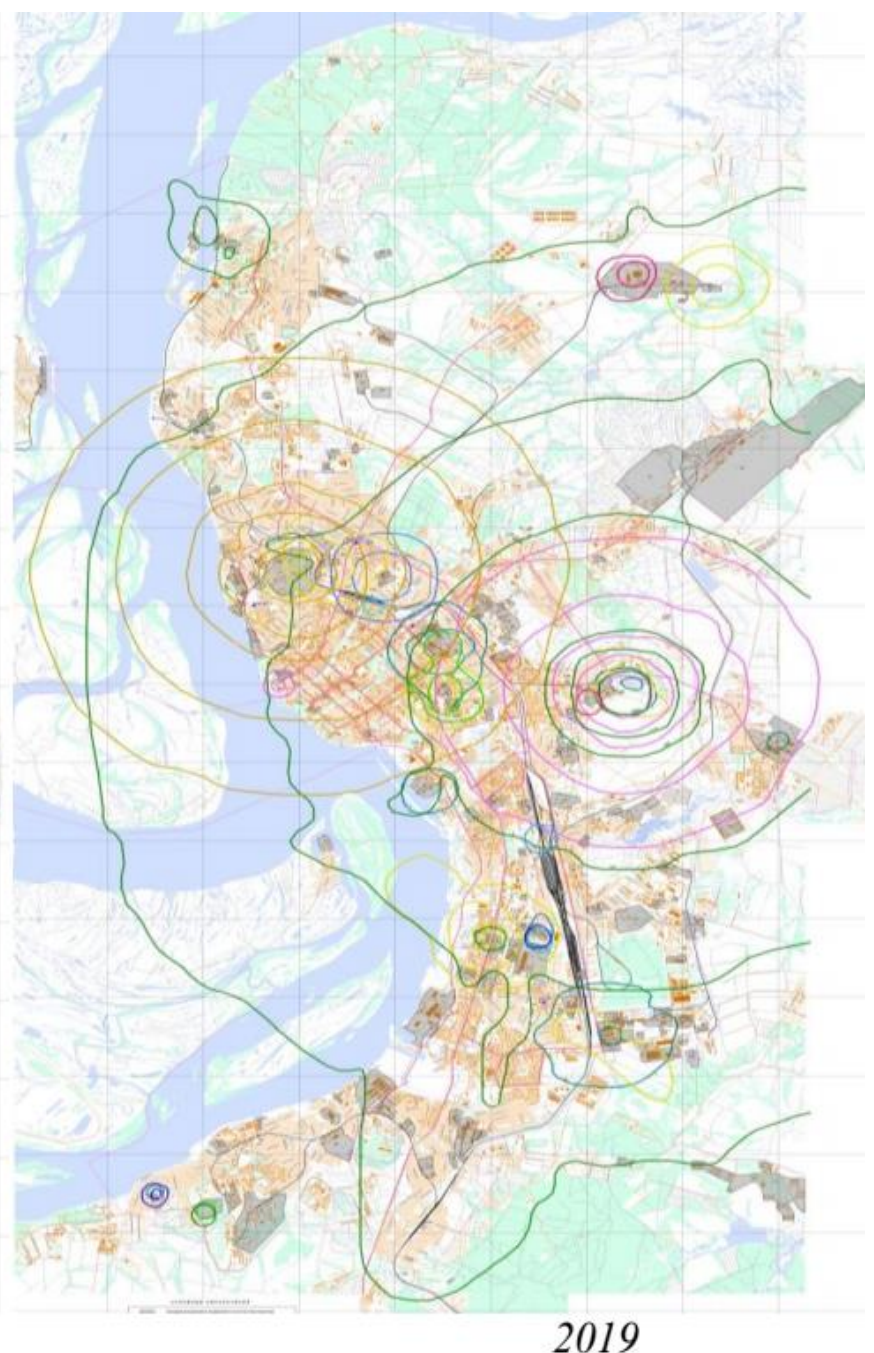

2019

Fig. 3. Maps of atmospheric air pollution by emissions from industrial enterprises current situation in 2003 and 2019 years

Figures 3 show that over 16 years, the level of air pollution in Khabarovsk has significantly decreased, while the number of suicide attempts has increased by 2.8 times. Moreover, the areas with the highest level of suicide (№12, №19, №34, №48) do not coincide with the zones of air, water, soil pollution, the presence of power lines, the proximity of roads and other environmental factors.

The medico-geographical studies are aimed not only at studying the patterns of the spread of suicides, but also to establish the causes of such patterns. This task, which belongs to the section of analytical epidemiology, often needs to be solved by special methods within the framework of population studies. In some cases, the analytical nature of such studies is associated with the study of the role of LIF in the formation of patterns of population distribution of suicides. These data demonstrate the fundamental possibility of predicting local changes in the health status of the population with various environmental changes. From the above facts, it 
follows that natural and anthropogenic factors do not affect the spread of suicides. MEGIS allows you to take into account and study the macrosocial, microsocial and social-psychological causes of DD and suicides. In order to clarify the role of microsocial factors in the emergence and during the course of depressive disorder that provokes suicide, we began by examining the composition of the family in which the adolescents lived. According to the data obtained, more than half $(60.0 \%)$ of adolescents lived in an incomplete family (a family without a father, with a father and step mother). Most of these teenagers regarded a similar situation as unsatisfactory, and frequent intra-family conflicts due to lack of secure attachment to the mother or stepfather.

In the course of further research, we found out that as the main factor in the onset of depressive disorder was parental pressure. The feeling of anxiety was especially pronounced in adolescents living with their own mother and stepfather $(32.0 \%)$ adolescents, in a family without a father $(24.0 \%)$ and in children house $(6.0 \%)$. Adolescents raised in an orphanage complained of pronounced feeling dissatisfaction and insecurity in connection with the lack of an environment of loved ones.

Only $24.0 \%$ of adolescents had a complete family, that is, they lived with both natural parents, and, as shown further research, were generally satisfied with the conditions of upbringing in family in a foster family or with relatives $8.0 \%$ of adolescents lived, but even in this case, they suffered from loneliness, since they were inherent some alienation, in their opinion, they did not feel emotional support from loved ones. adolescents who were raised by type of hypoprotection, most often were in a state of conflict in relations with their relatives $36.0 \%$. The reason they saw this conflict in material and everyday problems.

The conflict was perceived by them as hopeless, which contributed to the development chronic psychotraumatic situation and the emergence of a suicidal behavior. It is known that an important role in the formation of depressive disorders macrosocial factors also play a role. It should be noted that almost all adolescents of the first age period had a combined effect several macrosocial factors. In the second age period we identified significantly significant factors influencing the development of depressive disorders significantly more often in $36.0 \%$ of adolescents there were problems at school - "conflicts with the teacher" [Loginov et al., 2019].

It should be noted that among the cases of suicidal attempts, "paired" localizations (including at the place of residence, work, study) were not observed. According to our research, to macrosocial factors, formative depressive disorders in adolescents were conflicts with teachers, dissatisfaction with the organization and conditions of study, dissatisfaction with the degree of freedom and independence in society, fear of the uncertainty of your future; microsocial factors included difficulties in adapting in their interpersonal relationships with peers, more than half of the adolescents were in conflict relationships with members your family. This led to the onset of pre-suicide depressive disorders, provoked acute affective reactions and led to aggravation adaptation processes.

A similar circumstance requires the organization of general education psychological support institutions to provide assistance schoolchildren in a crisis situation. Time schedule for this services should be taken out of the educational process and can be implemented in individual and group trainings and consultations. Formation of personality traits, attitude, as to others, and to oneself, occurs during the learning process at school and further with mastering professional skills. Breakdown of mental and physical health reserves at each stage of the learning process can form adjustment disorder with acute and prolonged depressive reactions.

Thus, knowledge of the factors contributing to the formation depressive disorder turning into suicide creates an objective basis for developing a model preventive diagnostics based on the method of mathematical modeling. MEGIS allow you to take into account any qualitative and quantitative factors with a cartographic reference to the real area where suicide cases have been recorded (Fig. 4). 


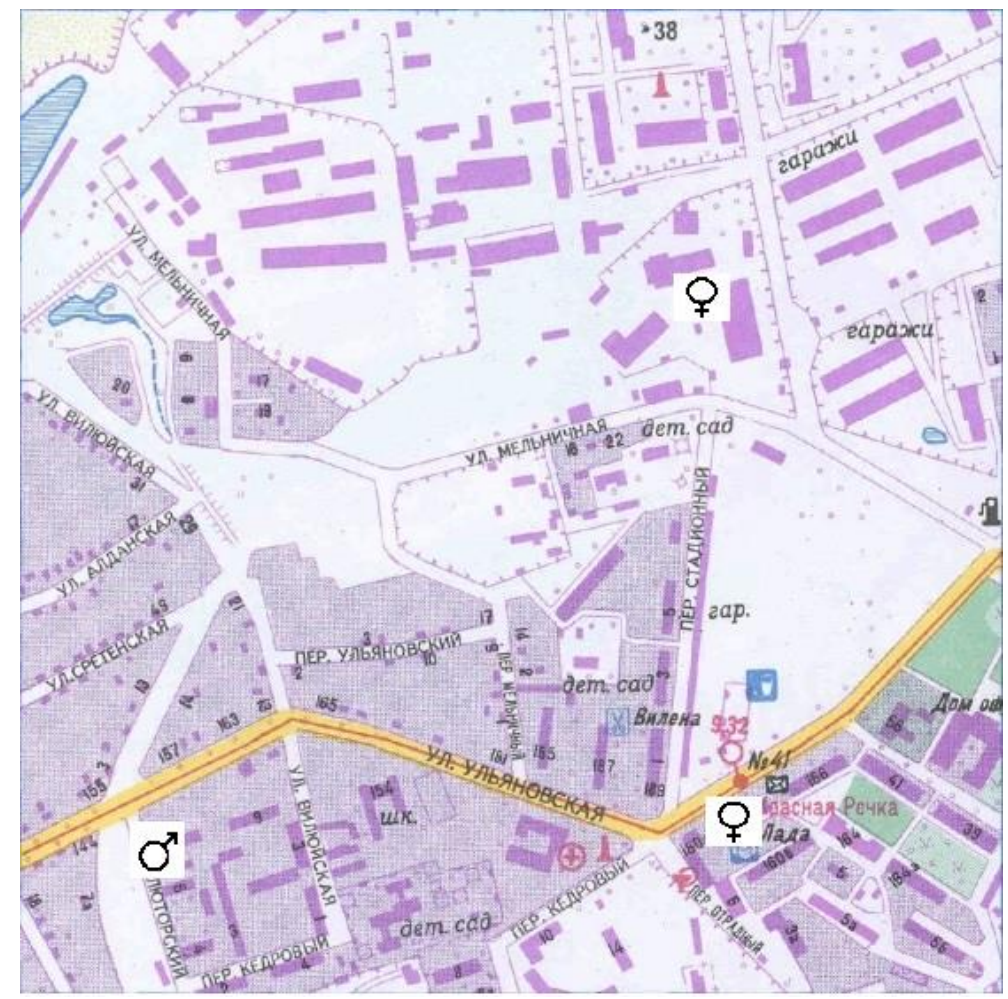

Fig. 4. localization by gender of suicide attempts in 48th square of Khabarovsk

The problems of combining and manipulating graphical and attribute data (gender, age, number of suicide attempts, composition of the family and other attributes) within a single integrated model that arise in medical and environmental studies are solved by means of tool packages of geographical information systems. MEGIS is based on a spatial model consisting of several thematic layers. Each graphic object in the layer has its own attribute description, which is stored in an embedded or external database. In this case, the layers of the spatial model can be any subsystems of a complex ecological object (system of objects). A methodology for creating geographic information systems in Khabarovsk has been developed, including data visualization on the suicide hazard of areas and location other harmful factors (Fig. 4.). The geographic information system of the city includes a map of the territorial distribution of cases of suicides, the most important socio-economic and medical-geographical characteristics of administrative districts. Methodological approaches to assessing the risk of suicides and DD, depending on the place of residence, have been developed. An appropriate software and hardware complex is being developed to solve the problems of medical ecology through MEGIS technologies and environmental monitoring. The possibility of applying a single methodological approach for geoinformation modeling of the spread of various forms of pre-suicide DD is justified.

\section{CONCLUSION}

MEGIS is an information recognition system based on the concept of information modeling, according to which all available information about the object of research is collected and accumulated, and then the recognition problem is solved. A feature of is the principles of organizing this information system allow to take into account the presence of a person on a sufficiently large territory and analyze the likelihood of its presence in different parts of a given territory. With the encouragement of MEGIS explored the influence of socio-psychological, socioecological and medical factors on the peculiarities of the territorial distribution of suicides, in- 
cluding advanced forms of pre-suicide DD, a population-epidemiological study was conducted in the Khabarovsk city. It was believed that the study of relationships between socio-economic and demographic and mental health characteristics of the individual administrative districts, on the one hand, and population frequency of a particular disease, on the other hand, can be an evidence of the social aspects of diseases.

The methodological basis of the study was the analysis of multiple linear regression with an assessment of the contributions of the studied factors to the formation of the output parameter. This original methodological approaches to the system analysis of non-psychotic depressive disorders in teenagers are considered. Are spent medico-psychological, socially-psychological, medico-epidemiological, ethnocultural researches of features of distribution of suicides and depressive disorders in Amur region of Khabarovsk territory. Despite certain successes in business of preventive diagnostics and treatment of depressive disorders and suicides, the problem of distribution of suicides remains to one of the major for medicine, psychology, sociology ecology and economy of Far East federal district. It is reliably established that mental disorders containing in their clinical manifestations a depressive symptom complex are the most suicidal, which was confirmed in our study.

It was revealed that the socio-economic and medico-demographic crises in Amur region are accompanied by an increase in the number of mental disorders, which contain in their clinical manifestations a depressive symptom complex, which is the most suicidal. The experience gained in Russia and abroad can be useful for a correct understanding of the social processes occurring in indigenous populations at the present stage of society development, and will help to develop a strategy for the prevention of suicide among indigenous peoples living in the Far East of the Khabarovsk Territory. It should be noted that the severity of depression and suicides in Russian Far East adolescents who made suicide attempts in our study often reached mild and moderate levels, and suicide was due to an inadequate type of personal response provoked by the use of alcohol or other surfactants. Significantly more often our teenagers committed suicide with adaptation disorder, short-term depressive and prolonged reaction. Mental problems in one family member cause a sense of shame and guilt among others.

Thus, for a long time, teenagers remain out of the psychiatrist's field of vision and do not receive specialized care, which leads to deterioration of the condition, up to a depressive episode of severe without psychotic symptoms. It is necessary to organize psychological support services at general educational institutions in the Amur Region to provide assistance to schoolchildren in a crisis situation. Knowledge of the causes and risk factors for suicidal behavior is necessary to develop preventive measures to prevent adolescents suicide. The time schedule for the work of this service should be outside the framework of the educational process and can be implemented through individual and group trainings and consultations. The result of preventive measures will be the preservation of the growing population of the Amur region of the Russian Far East.

\section{ACKNOWLEDGEMENTS}

The study was funded by the Russian Foundation of Basic Research. No 19-013-00018.

\section{REFERENCES}

1. Bostic J.Q., Rubin D.H., Prince J. Treatment of depression in children and adolescents. Journal of Psychiatric Practice. 2005. No 11(3). P. 141-154.

2. Carrellas N.W., Biederman J., Uchida M. How prevalent and morbid are subthreshold manifestations of major depression in adolescents? A literature review. J Affect Disord. 2017. V. 1. No 210. P. 166-173. 
3. Churkin A.A., Polozhiy B.S., Revenko V.I., Rakitsky G.F. Ethnocultural features of prevalence and clinical manifestations of mental disorders. Bulletin of Neurology, Psychiatry and Neurosurgery. 2009. No 2. P. 39-46 (in Russian).

4. Clayton K.M., Stewart S.M., Wiebe D.J., McConnel C.E., Hughes C.W., White P.C. Maternal depressive symptoms predict adolescent healthcare utilization and charges in youth with type 1 diabetesm. Health Psychology. 2013. No 32(9). P. 1013-1022.

5. Demcheva N.K., Yazdovskaya A.V. Overall incidence of mental disorders in the Russian Federation and federal districts in 2017-2019. Bulletin of Neurology, Psychiatry and Neurosurgery. 2021. No 1. P. 64-59 (in Russian).

6. Gladstone T.R.G., Beardslee W.R. The prevention of depression in children and adolescents: a review. Can. J Psychiatry. 2009. No 54(4). P. 212-21. DOI: 10.1177/070 674370905400402.

7. Jinnin R., Okamoto Y., Takagaki K. et al. Detailed course of depressive symptoms and risk for developing depression in late adolescents with subthreshold depression: a cohort study. Neuropsychiatric Disease and Treatment. 2016. V. 13. P. 25-33.

8. Kosykh N.E., Lopatin A.S., Novikova O.Yu., Savin S.Z. Geoinformation systems in problems of medical ecology. Vladivostok: Dalnauka, 2008. 152 p. (in Russian).

9. Krasnov V.N. Depression as a social and clinical problem of modern medicine. Russian psychiatric journal. 2011. No 6. P. 8-10 (in Russian).

10. Kretinin D.F., Samokhin D.V., Goncharova E.M. Suicidal behavior in the structure of depressive disorders in young people. Siberian Bulletin of psychiatry and narcology. 2015, № 1(86). P. 9-15 (in Russian).

11. Kripke D.F., Lesse S. The multivariant masks of depression. Am. J. Psychiatry. 1998. V. 124(1). P. 35-40.

12. Loginov I.P., Savin S.Z. Geoinformation systems in the population analysis of the distribution of depressive disorders in Khabarovsk. InterCarto. Intergis. 2020. T. 26. No 3. P. 39-52 (in Russian).

13. Loginov I.P., Savin S.Z., Solodkaya E.V. Comparative analysis of the spread of depressive disorders in adolescents in Russia and China. Mental Health of Children and Adolescent. 2019. No 3. P. 4-12 (in Russian).

14. Malone K.M., Kelleher C., Kuch G., et al. Suicidal ideation is associated with elevated inflammation in patients with major depressive disorder. Depress. Anxiety. 2013. V. 30(4). P. 307-14.

15. Oorzak L.N. Problem of youth suicide in the republic of Tuva. Bulletin of Tuva State University. Pedagogical sciences. 2012. No 4. P. 31-36 (in Russian).

16. Polumienko S.K., Savin S.Z., Turkov S.L. Information models and decision-making methods in regional ecological and economic systems. Vladivostok: Dalnauka, 2007. 376 p. (in Russian).

17. Shipitsyna L.M., Ivanov E.S., Stetsenko E.M. Motives of suicidal behavior in adolescents. Russian psychiatric journal. 2000, V. 5. P. 40-45 (in Russian).

18. Stewart S.M., Kennard B.D., Lee P.W.H., Mayes T., Hughes C.W., Emslie G. Hopelessness and suicidal ideation among adolescents in two cultures. Journal of Child Psychology, Psychiatry and Allied Disciplines. 2010. V. 46. P. 364-372.

19. Syrokvashina K.V. Modern psychological models of suicidal behavior in adolescence. Counseling psychology and psychotherapy. 2017. No 25(3). P. 60-75.

20. Wasserman D., Zalsman G., Hawton K. Suicide prevention strategies revisited: 10-year systematic review. Lancet Psychiatry. 2016. No 3(7). P. 646-59. 